\title{
Dual-Cavity Dual-Output Multi-Wavelength Fiber Laser Based on Nonlinear Polarization Rotation Effect ${ }^{1}$
}

\author{
S. J. Tan ${ }^{a}$, S. W. Harun ${ }^{a, b}, *$, N. S. Shahabuddin ${ }^{a}$, H. Arof ${ }^{a}$, and H. Ahmad ${ }^{b}$ \\ ${ }^{a}$ Department of Electrical Engineering, Faculty of Engineering, University of Malaya, 50603 Kuala Lumpur, Malaysia \\ ${ }^{b}$ Photonics Research Center, University of Malaya, 50603 Kuala Lumpur, Malaysia \\ *e-mail: swharun@um.edu.my \\ Received April 21, 2012; in final form, May 27, 2012; published online September 3, 2012
}

\begin{abstract}
We demonstrate a multi-wavelength fiber laser based on nonlinear polarization rotation (NPR) in dual-cavity configuration with two output ports. The laser employs a piece of erbium doped fiber (EDF) and semiconductor optical amplifier (SOA) as the gain medium in a separate cavity. By incorporating PCF in the dual cavity the non-linear polarization rotation (NPR) effect is enhanced and thus higher output and more oscillating lasing can be achieved. The laser produces three strong lines with a spacing of $2.40 \mathrm{~nm}$ and side mode suppression ratio (SMSR) of more than $10 \mathrm{~dB}$ at $1535 \mathrm{~nm}$ region. Another output produces 8 lines of optical comb with a spacing of 0.54 at $1570 \mathrm{~nm}$ region.
\end{abstract}

DOI: $10.1134 / \mathrm{S} 1054660 \mathrm{X} 12100222$

\section{INTRODUCTION}

Multi-wavelength fiber lasers are widely being used in applications such as in dense wavelength division multiplexing (DWDM), optical fiber sensor system, optical testing and spectroscopy [1-3]. Various gain media have been utilized for the multi-wavelength generation and they include erbium-doped fiber amplifier (EDFA) [4], semiconductor optical amplifier [5-8] and Raman amplifier [9] in different configurations such as ring, linear or figure of eight cavity structures. Multi-wavelength erbium doped fiber lasers (EDFL) are favorable as they have high saturation power and low threshold. However, EDFLs suffer from strong mode competition, which causes unstable lasing. To overcome this problem, many methods have been proposed such as by using semiconductor optical amplifier (SOA) which exhibit inhomogeneous broadening property, utilizing FWM effect [10, 11], using twin core EDF [12] and many more.

Lately, nonlinear polarization rotation (NPR) effect in optical fibers has also been used to demonstrate multi-wavelength fiber lasers [13, 14]. The NPR produces intensity dependent loss in the cavity, which then contributes to phase shifting and multi-wavelength signal generation. Recently, many works focus on compact multi-wavelength lasers with a short nonlinear gain medium and therefore an interest on a nonlinear photonics crystal fiber (PCF) is tremendously increased. However, the cost of this fiber is reasonably high compared to the standard single mode fiber (SMF). The dual output multi-wavelength laser is also gaining interest for special applications in optical communication and sensors. To date, NPR-based multi-wavelength fiber laser is only demonstrated in a single ring cavity. In this

\footnotetext{
${ }^{1}$ The article is published in the original.
}

paper, we demonstrate for the first time NPR-based multi-wavelength fiber laser using hybrid gain media of EDF and SOA in bidirectional dual-cavity structure which sharing the same non-linear fiber, PCF. This new approach is able to generate multi-wavelength in $\mathrm{C}$ - and L-band regions owing to the different operating regions of SOA and EDF. In addition, the wavelength spacing can be changed by controlling the birefringence, intra-cavity power and loss performances inside the cavity. The new dual-cavity design increases the compactness and reduces the cost of the laser since it is sharing most of the main components.

\section{EXPERIMENTAL SETUP}

The experimental set-up of the proposed scheme for dual-cavity multi-wavelength fiber laser is illustrated in Fig. 1. This configuration consists of two gain media (50 m long EDF and SOA), two optical circulators $(\mathrm{OC} 1$ and $\mathrm{OC} 2)$, two $10 \mathrm{~dB}$ couplers $(\mathrm{C} 1$ and $\mathrm{C} 2)$ and one piece of photonic crystal fiber (PCF). The $\mathrm{EDF}$ has an erbium ion concentration of $440 \mathrm{ppm}$, cut-off wavelength of $950 \mathrm{~nm}$ and absorption rate of $5.5 \mathrm{~dB} / \mathrm{m}$ at $980 \mathrm{~nm}$. It is pumped by $1480 / 1550 \mathrm{~nm}$ wavelength-division multiplexer (WDM) to provide amplification in the L-band region. The SOA used is made from an InGaAsP-InP ridge waveguide with facets angled at $10^{\circ}$ and coated with anti-reflection film. It has a saturated output power of $10 \mathrm{dBm}$ at the maximum bias current of $300 \mathrm{~mA}$ and a center operating wavelength of $1534 \mathrm{~nm}$ with a spectral width of $40 \mathrm{~nm}$. The SOA has a small signal gain of $20 \mathrm{~dB}$ at a gain saturation current of $160 \mathrm{~mA}$. The PCF is $50 \mathrm{~m}$ long with a solid core structure and a nonlinear coefficient of about $11 \mathrm{~W}^{-1} \mathrm{~km}^{-1}$. It has a zero-dispersion wavelength of around $1550 \mathrm{~nm}$ and an attenuation of 